\title{
Cuando la salud sexual y (no) reproductiva es objetada a conciencia. Un análisis desde la teoría política y el enfoque de derechos
} When sexual and (non-) reproductive health is objected to consciousness. An analysis from the political theory and the rights approach

\author{
María Julieta Cena \\ Universidad Nacional de Córdoba (Argentina) \\ ORCID ID 0000-0003-4333-1451 \\ mjulietacena@gmail.com
}
Cita recomendada:
Cena, M. J. (2021). Cuando la salud sexual y (no) reproductiva es objetada a conciencia. Un análisis desde la teoría política y el enfoque de derechos. Eunomía. Revista en Cultura de la Legalidad, 20, pp. 172-185.
doi: https://doi.org/10.20318/eunomia.2021.6068

Recibido / received: 14/01/2020 Aceptado / accepted: 24/07/2020

\section{Resumen}

El presente trabajo aborda la tensión entre la salud sexual y (no) reproductiva y la objeción de conciencia cuando es ejercida ante interrupciones legales de embarazos, desde una perspectiva crítica feminista con enfoque de derechos. A tales fines, el análisis gira en torno al Estado de derecho como escenario político donde convergen las fuerzas antagónicas de los movimientos feministas y las resistencias heteropatriarcales religiosas. Para ello, se visibiliza a la salud sexual y (no) reproductiva en el marco de los derechos humanos, así como las particularidades de la objeción de conciencia en dicho terreno, sus consecuencias y efectos en términos de democracia e igualdad.
\end{abstract}

\section{Palabras clave}

Objeción de conciencia, salud sexual y no reproductiva, interrupciones legales de embarazos, Estado de derecho, democracia, igualdad, derechos humanos, derechos sexuales y reproductivos. 


\begin{abstract}
This work deals with the tension between sexuality and non-reproduction health and conscientious objection when it is exercised in the face of legal interruptions of pregnancies, from a feminist critical perspective with a rights focus. For this purpose, the analysis revolves around the rule of law as a political scenario where antagonistic forces typical of the struggles of feminist movements and hetero-patriarchal religious resistance converge. Sexual and (non-) reproductive health is made visible in the framework of human rights and the particularities of conscientious objection in this field, as well as its consequences and effects in terms of democracy and equality.
\end{abstract}

\title{
Keywords
}

Consciousness objection, sexual and non-reproductive health, legal interruptions of pregnancy, rule of law, democracy, equality, human rights, sexual and reproductive rights.

\begin{abstract}
SUMARIO. 1. Introducción. 2. La salud sexual y no reproductiva: definiciones desde un enfoque de derechos humanos. 3. Objeción de conciencia y el deber de obediencia en casos de salud sexual y no reproductiva. 4. La objeción de conciencia ante la salud sexual y no reproductiva: religión, Estado y movimientos sociales. 5. Algunas consideraciones sobre el alcance de la objeción de conciencia frente a la salud sexual y no reproductiva en términos de democracia e igualdad. 6. Reflexiones finales.
\end{abstract}

\section{Introducción}

La objeción de conciencia (en adelante OC) ha permitido en distintos momentos históricos, abstenerse de cumplir diversas obligaciones legales como acto de resistencia a las relaciones de poder hegemónicas (Puga y Vaggione, 2015, p. 94). Tradicionalmente, y en contextos de respeto al pluralismo, se ha presentado como una herramienta democrática que permite la convivencia de sectores de cosmovisiones éticas diferentes. Sin embargo, frente a los derechos sexuales y reproductivos (DSR), en general, y la salud sexual y (no) reproductiva (SS/SR), en particular, la figura se complejiza y obliga a su revisión.

Es menester destacar que la OC toma relevancia inscrita en un Estado de Derecho democrático ${ }^{1}$ donde el imperio de la ley, como expresión de la voluntad popular, exige la sumisión a ella de todos los poderes: estatales, no estatales, sociales, económicos, religiosos, etc., (Díaz, 2010, pp. 25-32). No obstante, y paradójicamente, mediante la OC los ordenamientos han posibilitado incumplimientos normativos en aras de la protección de derechos individuales.

Tradicionalmente, la OC fue justificada como un mecanismo que protegía al pluralismo y las disidencias ante los criterios mayoritarios consagrados en las leyes, sobre todo aquellas que legislaban sobre el servicio militar y la guerra. Con el tiempo, a la par que se debilitaba la utilización de la figura en dicho terreno, la figura fue

\footnotetext{
${ }^{1}$ El presente trabajo girará en torno a la OC en el marco del Estado de Derecho democrático por entender que la problemática es trascendente en dicho sistema socio-político legal. Sin embargo, a los fines de ejemplificar la manera en que la cuestión ha sido planteada frente a políticas de reivindicación e institucionalización de derechos de salud sexual y (no) reproductiva, en algunas oportunidades, haré referencia a situaciones específicas de ciertos países, conjugando así un análisis teórico-práctico.
} 
ganando fuerza en el ámbito sanitario, principalmente ante interrupciones legales de embarazos $^{2}$ (en adelante ILE) (Triviño Caballero, 2018, p.199).

Por su parte, los DSR, en el marco de los derechos humanos (DH), supone entenderlos como aquellos que comprenden tanto derechos referidos al libre ejercicio de la sexualidad, como a la autodeterminación y a la reproducción sexual (Richardson, 2000, pp. 105-135; Peñas Defago, 2012, pp. 196-201). Cierto es que, en los ordenamientos jurídicos estatales, se pueden encontrar legislaciones relativas a derechos vinculados con la "reproducción" (por ejemplo, leyes sobre fertilizaciones asistidas, partos respetados, seguridad durante el embarazo, etc.), como a la "no reproducción" (anticoncepción, reasignación genital por identidad de género, contracepción quirúrgica, etc.). Estos últimos, y sobre todo las ILE, al escindir la sexualidad de la reproducción y cuestionar los roles de sexo genéricos, producen fuertes resistencias por parte de los sectores heteropatriarcales religiosos ${ }^{3}$.

Si bien los DSR importan una amplia gama de derechos vinculados con el respeto a la dignidad humana (tales como la igualdad, la educación sexual, el derecho a una vida libre de violencia, entre otros), a los fines del presente análisis me detendré en las tensiones de la OC cuando es interpuesta ante derechos de SS/SR, particularmente ante ILE. Tal especificidad responde al entendimiento de que, en el entramado de los DSR, las ILE cuestionan el control de los cuerpos gestantes inscrita en la SS/SR como DH.

A medida que los Estado legalizan las interrupciones de embarazos, sectores conservadores heteropatriarcales con fuerte impronta religiosa arbitran múltiples mecanismos de resistencia entre los que se destacan la OC (Cena, 2020, pp. 149160; Savulescu, 2006, pp. 294-297; Vaggione y Puga, 2015, pp. 104-105). En ese marco, la OC ha abandonado el alcance tradicional y tomado una extensión significativa que, conjugado con el poder e influencia de los/as profesionales de la salud, potencia la posibilidad de que su implementación desarticule políticas públicas de acceso a la SS/SR (Puga y Vaggione, 2015, pp. 104-105; Alegre, 2009, pp. 21-22; Cena, 2020, pp.149-160). A esto debe agregarse la situación de desigualdad en el vínculo personal sanitario y usuario/a del sistema de salud, así como el deber relacional ${ }^{4}$ de los/as profesionales de la salud, en tanto actores imprescindibles para las realizaciones de las prácticas relativas a SS/SR en el marco de los sistemas institucionales.

\footnotetext{
2 Utilizaré el término "interrupciones legales de embarazos" por entender que el mismo nuclea los abortos reconocidos normativamente por los ordenamientos jurídicos, más allá del sistema o modalidad con el que se los permita (abortos por causales, interrupciones voluntarias, etc.). Para mayor información sobre los sistemas y contextos recomiendo la lectura, a la que, por razones de brevedad me remito, de Bergallo, P. (2011). Introducción. La liberalización del aborto: contextos, modelos regulatorios y argumentos para su debate. En P. Bergallo (comp.), Aborto y justicia reproductiva (pp. 1-54). Buenos Aires, Argentina: Ed. del Puerto.

${ }^{3}$ La denominación intenta nuclear la heterogeneidad de características que guarda el activismo de los sectores opuestos a los DSR. En particular, sobre la OC, cabe aclarar que las convicciones que se aluden para la abstención de ILE están vinculadas a concepciones sobre la vida y el biopoder médico íntimamente ligados a lo religioso, más allá de la impronta secular. Para más información sobre la propuesta conceptual "activismo heteropatriarcal" recomiendo la lectura de Morán Faúndes, J. M. (2018). Religión, secularidad y activismo heteropatriarcal: ¿qué sabemos del activismo opositor a los derechos sexuales y reproductivos en Latinoamérica? La ventana. Revista de estudios de género, 5(47), 97-138. Recuperado de http://www.scielo.org.mx/scielo.php?script=sci arttext\&pid=S1405-9436201800 $0100097 \&$ Ing=es\&tIng=es

4 Tal y como profundizaré más adelante, los "deberes relacionales" generan obligaciones en virtud de una determinada relación personal. En el caso de la OC en el ámbito sanitario, el vínculo surge en virtud del ejercicio de una profesión u oficio. Para mayor información sobre el concepto, recomiendo la lectura de Gardner, J. (2018). From personal life to private law. Oxford, Reino Unido: Oxford University Press.
} 
En este entramado el Estado cumple un rol trascendental como actor político de las acciones y decisiones necesarias para la consumación de la igualdad. Lo expuesto, en tanto por un lado reconoce el DH a la SS/SR mediante la legalización de las interrupciones de embarazos y, por el otro, permite que las personas obligadas se abstengan de cumplir la orden legal bajo el halo de objeciones de conciencias de amplia interpretación. En otras palabras, cuando el Estado, por acción u omisión, genera posibilidades de acción de la OC como una opción y no como un recurso excepcional incumple los compromisos democráticos de igualdad.

Por estas razones, en el presente trabajo analizaré la tensión existente entre la OC y las ILE (como derecho de SS/SR) desde un enfoque de DH que posiciona a los Estados como garantes de las obligaciones internacionalmente contraídas (Etchichury, 2015, pp. 13-15; Peñas Defago, 2012, p. 197). Concibo pues, que el Derecho debe ser entendido como un discurso social que en su interrelación dota de sentido las conductas y convierte en sujetos a quienes lo practican. $Y$, además, tiene la particularidad de detentar una función paradojal: por un lado, legitima las relaciones de poder $y$, por el otro, goza de potencialidad como un instrumento para la transformación (Ruiz, 2003, p.1).

2. La salud sexual y no reproductiva: definiciones desde un enfoque de derechos humanos

La noción DSR condensa procesos sociales, políticos y normativos (Petracci y Pecheny, 2007, p. 19) de reciente institucionalización, con fuerte participación y acción de los movimientos por los $\mathrm{DH}$, la diversidad sexual y los feminismos. Así, los DSR están conformados por un conjunto de derechos enunciados en los tratados internacionales que, a partir de la década de los noventa, comienzan a incorporarse lentamente en el discurso de los $\mathrm{DH}$ (Vaggione, 2012, pp. 47-49) y en las legislaciones internas de los Estados.

En el año 1994, en Egipto, se llevó a cabo la Conferencia Internacional sobre Población y Desarrollo: El Cairo (CIPD) ${ }^{5}$, mediante la cual la comunidad internacional reconoció formalmente las desventajas y desigualdades sexo-genéricas perpetuadas por las estructuras, centrándose en una concepción de derechos más allá de los aspectos demográficos. Dentro de ese marco se desarrollaron importantes definiciones para las luchas por la igualdad de género, los DSR, las acciones para mejorar la situación de las niñas, el estatus de la mujer, la situación de los adolescentes, etc.

Las recomendaciones producto de esta conferencia conformaron lo que se ha denominado «Programa de acción». Este programa enfatiza tanto el derecho a la libre decisión como la necesidad de eliminar toda forma de coacción en relación a la salud como componentes básicos para garantizar la SS/SR de la población. De esta manera, se definió la salud reproductiva como un estado de bienestar general tanto físico como mental y social, y no de mera ausencia de enfermedades o dolencias vinculadas con el sistema reproductivo, funciones y procesos.

Tal como enunciara al comienzo, la salud reproductiva entraña la capacidad de disfrutar de una vida sexual satisfactoria y sin riesgos, así como la libertad para decidir procrear o no, cuándo y con qué frecuencia (CIPD, 1994, p. 37). En efecto, la salud sexual se proyecta en «...el desarrollo de la vida y de las relaciones personales y no meramente en el asesoramiento en materia de reproducción y enfermedades de

\footnotetext{
${ }^{5}$ Naciones Unidas. (1994, septiembre). Informe de la Conferencia Internacional sobre la Población y el Desarrollo. Recuperado de https://www.unfpa.org/sites/default/files/pub-pdf/icpd spa.pdf.
} 
transmisión sexual» (CIPD, 1994, p. 37). Asimismo, la atención y ejercicio de la SS/SR se traduce en el conjunto de métodos, técnicas y servicios que contribuyan a evitar y resolver la afectación de estos derechos.

En cuanto al sistema universal de $\mathrm{DH}$, el Comité de Derechos Económicos, Sociales y Culturales (Comité DESC), en el año 2016, emitió la Observación General $\mathrm{N}^{\circ} 22^{6}$, mediante la cual ratifica que la SS/SR forma parte del derecho a la salud consagrado en el artículo 12 del Pacto Internacional de Derechos Económicos, Sociales y Culturales (PIDESC). Por consiguiente, los Estados partes del PIDESC deben garantizar que toda persona disfrute del más alto nivel posible de salud física y mental, incluida la SS/SR.

La igualdad a la luz del derecho a decidir sobre la sexualidad y la reproducción o no, exige la visibilización de las desigualdades sociales, económicas y políticas de las mujeres y personas gestantes ${ }^{7}$ en relación a los hombres. Corresponde entonces revisar el discurso de los $\mathrm{DH}$ desde el reconocimiento de la diversidad y los feminismos, así como de los contextos sociales y culturales (Correa y Petchesky, 1995 , p. 117), en tanto las mujeres y personas gestantes no tienen las mismas posibilidades de decisión a causa de las relaciones de poder que permean la vida, reproducidas y perpetuadas por las estructuras y los activismos de los sectores heteropatriarcales religiosos.

El estatus de legalidad de las interrupciones de embarazos es crucial para la salud y los $\mathrm{DH}$ de las mujeres y personas gestantes, así como también el acceso a prácticas seguras y eficaces, para lo cual el Estado debe arbitrar legislaciones y políticas públicas adecuadas. De esta forma, las ILE, como parte de la SS/SR, plantean la reivindicación de una verdadera igualdad y acceso a la ciudadanía, susceptible de ser obstaculizada si no están dadas las condiciones necesarias y disponibles los recursos materiales humanos y simbólicos para el conjunto de individuos y grupos que forman la comunidad política de cada sociedad (Petracci y Pecheny, 2007, pp. 32-37). Dentro de ellos, se inscribe la capacidad de personal sanitario disponible para la atención permanente de las ILE.

3. Objeción de conciencia y el deber de obediencia en casos de salud sexual y no reproductiva

La doctrina ha conceptualizado clásicamente la OC como:

Aquel incumplimiento de un deber jurídico motivado por la existencia de un dictamen de conciencia que impide observar el comportamiento prescrito y cuya finalidad se agota en la defensa de la moralidad individual, renunciando a cualquier estrategia de cambio político o de búsqueda de adhesiones (Gascón Abellán, 1990, p. 85).

En este sentido, la OC se presenta como una clase de desobediencia al Derecho (Gascón Abellán, 1990, pp. 27-40; Pietro Sanchis, 2011, p. 981) que goza de resguardo jurídico al sortear las sanciones correspondientes por incumplimiento a

\footnotetext{
${ }^{6}$ Naciones Unidas. (2008, mayo). Observaciones generales aprobadas por el Comité de Derechos Económicos, Sociales y Culturales. Recuperado de https://confdts1.unog.ch/1\%20SPA/Tradutek/Derechos hum Base/CESCR/00 1 obs grales Cte\%20Dchos\%20 Ec\%20Soc\%20Cult.html

${ }^{7}$ Usaré este término para referir tanto a los derechos de las mujeres como a los de otras identidades LGTBIQ+ que tengan posibilidad de gestar y, por consiguiente, se encuentren en condiciones de requerir ILE.
} 
la ley. Tal es, que las primeras incorporaciones a los ordenamientos jurídicos fueron asociadas a la abstención del servicio militar obligatorio sin condenas por infracción.

La potencia de las luchas que confrontaban el poder del Estado en democracias liberales promovió el reconocimiento explícito de la OC como un derecho, llegando incluso a consignarse en algunas constituciones nacionales. Actualmente, en el mundo occidental, siete cartas magnas refieren a la figura de manera expresa, principalmente ligado al servicio militar. Ellas son las constituciones de España, Rumania, Portugal, Ecuador, Paraguay, Cuba y Venezuela ${ }^{8}$.

Históricamente la doctrina diferenció la OC de la desobediencia civil, por determinar que aquella no debería apelar a un sentido de justicia, sino limitarse a no cumplir con una obligación legal por considerar que no existen bases para una compresión mutua. Si bien los fundamentos para la abstención al servicio militar obligatorio giraron en torno a motivos políticos y/o religiosos ${ }^{9}$, su migración hacia el sistema sanitario estuvo fuertemente vinculado a convicciones heteropatriarcales de base religiosa motivadas por el reconocimiento de la SS/SR como derecho. En efecto, a la par de la legalización de las interrupciones de embarazos, la agenda jurídica también extendió el uso de la OC al personal sanitario. Tanto es así que, en muchas oportunidades, las licencias a no cumplir con las obligaciones profesionales en el ámbito de las ILE, surgen de las mismas normas que reconocen el derecho a la interrupción.

Se diría pues que ciertos sectores heteropatriarcales religiosos contrarios a los DSR, a través de la OC, adoptan una retórica secular ${ }^{10}$ que les permite sostener las

\footnotetext{
${ }^{8}$ Constitución de Cuba. Título V. Capítulo II. Artículo 54: «La objeción de conciencia no puede invocarse con el propósito de evadir el cumplimiento de la ley o impedir a otro su cumplimiento o el ejercicio de sus derechos».

Constitución de Ecuador. Título II. Capítulo 6. Artículo 66: «Se reconoce y garantizará a las personas: (...) El derecho a la objeción de conciencia, que no podrá menoscabar otros derechos, ni causar daño a las personas o a la naturaleza».

Constitución de España. Título I. Capítulo Segundo. Artículo 30.2.: «La ley fijará las obligaciones militares de los españoles y regulará, con las debidas garantías, la objeción de conciencia, así como las demás causas de exención del servicio militar obligatorio, pudiendo imponer, en su caso, una prestación social sustitutoria». Título I. Capítulo Cuarto. Artículo 53.2.: «Cualquier ciudadano podrá recabar la tutela de las libertades y derechos reconocidos en el artículo 14 y la Sección primera del Capítulo segundo ante los Tribunales ordinarios por un procedimiento basado en los principios de preferencia y sumariedad y, en su caso, a través del recurso de amparo ante el Tribunal Constitucional. Este último recurso será aplicable a la objeción de conciencia reconocida en el artículo $30 »$.

Constitución de Paraguay. Parte I. Título II. Capítulo II. Artículo 37: «Se reconoce la objeción de conciencia por razones éticas o religiosas para los casos en que esta Constitución y la ley la admitan». Parte I. Título II. Capítulo XI. Artículo 129: «Quienes declaren su objeción de conciencia prestarán servicio en beneficio de la población civil, a través de centros asistenciales designados por ley y bajo jurisdicción civil. La reglamentación y el ejercicio de este derecho no deberán tener carácter punitivo ni impondrán gravámenes superiores a los establecidos para el servicio militar».

Constitución de la República Portuguesa. Parte I. Título II. Capítulo I. Artículo 41.6.: «Se reconoce el derecho a la objeción de conciencia, en los términos que establezca la ley».

Constitución de Rumania. Título II. Capítulo II. Artículo 42.2.: «No constituye trabajo forzoso: a) El servicio militar, así como las actividades sustitutorias realizadas, de acuerdo a la ley, por motivos religiosos o de objeción de conciencia».

Constitución de la República Bolivariana de Venezuela. Título III. Capítulo III. Artículo 61: «Toda persona tiene derecho a la libertad de conciencia y a manifestarla, salvo que su práctica afecte la personalidad o constituya delito. La objeción de conciencia no puede invocarse para eludir el cumplimiento de la ley o impedir a otros su cumplimiento o el ejercicio de sus derechos».

${ }^{9}$ Para mayor información sobre los fundamentos de las objeciones a otras obligaciones, por cuestiones de brevedad, recomiendo la lectura de Triviño Caballero, R. (2014). El peso de la conciencia: la objeción en el ejercicio de las profesiones sanitarias. Madrid, España: CSIC.

${ }^{10}$ Para más profundidad sobre la secularización de las estrategias religiosas recomiendo las lecturas de Mujica, J. (2007). Economía Política del Cuerpo. La reestructuración de los grupos conservadores y el biopoder. Lima, Perú: Centro de Promoción y Defensa de los Derechos Sexuales y Reproductivos;
} 
situaciones de poder y control sobre la sexualidad y la reproducción. Por consiguiente, pugnan por una $\mathrm{OC}$ reconocida como un derecho irrestricto y con un amplio alcance. Desde esta premisa, la OC es ejercida como estrategia jurídica susceptible de desarticular políticas públicas destinadas al ejercicio de los DSR (Ariza Navarrete, 2012, p. 26).

Si seguimos el criterio tradicional analizado de la OC y, por consiguiente, sostenemos que la obediencia de las normas es un problema para las minorías (Rawls, 1971, pp. 405-410), la desobediencia tiene otro alcance cuando proviene de sectores de poder mayoritarios en términos cualitativos. Lo expuesto nos ocupa en tanto que la capacidad política para generar legislación de quienes objetan prácticas de ILE los/as sitúa más cerca de las mayorías que de las minorías en sentido posicional y relacional (Puga y Vaggione, 2015, p. 114). Es que, al contrario de lo que sucedía cuando se reclamaba la abstención de obligaciones al servicio militar, en la $\mathrm{OC}$ en el terreno de la SS/SR confluyen el poder médico con convicciones de fuerte impronta religiosa (Alegre, 2009, pp. 15-16; Cena, 2020, pp. 149-176), que, en contextos sociopolíticos de alta permeabilidad a las posiciones religiosas, tienen alto impacto e influencia en la toma de decisiones. Estas circunstancias nos plantean la necesidad de cuestionarnos si la OC, en la actualidad, frente a casos de SS/SR, configura las condiciones tradicionalmente desarrolladas para su procedencia y, si en este sentido, es dable todavía entenderla como un acto que aporta a la democracia y cuáles son las estrategias estatales al respecto.

La extensión que la figura tiene en la actualidad ante las ILE genera inclusiones de conflictos que se asemejan más a desobediencias civiles con fuerte identificación a posicionamientos religiosos. De hecho, quienes defienden una noción amplia de la OC rechazan el apego a sus concepciones tradicionales por entender que ello obturaría un supuesto devenir histórico de la objeción, recomendando la implementación de la designación plural "objeciones de conciencias" (Triviño, 2018, pp. 200-203) como señal de multiplicidad y generalidad.

4. La objeción de conciencia ante la salud sexual y no reproductiva: religión, Estado y movimientos sociales

La influencia de los movimientos feministas en el discurso de los $\mathrm{DH}$ logró mayor visibilización y debate en torno a los DSR, inaugurando una nueva etapa sobre su discusión jurídica (Vaggione, 2012, pp. 45-48). Estos movimientos feministas reclaman reconocimientos legales y políticos con impacto en la sociedad en su conjunto y no solo en el colectivo representado, lo que en términos de Offe (1992, pp. 163-188) daría a llamarse «nuevos movimientos sociales».

En particular, los movimientos feministas se han caracterizado por instar la satisfacción de los $\mathrm{DH}$ mediante la protección de la identidad, dignidad y el tratamiento equitativo (Offe, 1992, pp. 163-188). Por consiguiente, la articulación del concepto de $\mathrm{DH}$ con derechos de la mujer y otras identidades, así como de autonomía e integridad física y psíquica con SS/SR, obliga a los Estados de Derecho democráticos a la promoción y facilitación de transformaciones en los sistemas de justicia, de salud, de educación, de policía, entre otras (Montaño, 1996, p. 8). En este sentido, los

\footnotetext{
Vaggione, J.M. (2012). La "cultura de la vida". Desplazamientos estratégicos del activismo católico conservador frente a los derechos sexuales y reproductivos. Religião e Sociedade, 32(2), 57-80; y Vaggione, J.M. (2013). Laicidad y sexualidad. En P. Salazar y P. Capdevielle (coords.), Colección "Jorge Carpizo". Para entender y pensar la Laicidad (pp. 435-440). México D.C., México: UNAM y IIDC.
} 
movimientos feministas son actores con capacidad para transformar las estructuras sociales a través de la reflexión y la identificación (Jessop, 2014, pp. 1-17).

Sin embargo, pese a los avances legislativos, persisten obstáculos institucionales, políticos y simbólicos que manifiestan la resistencia a los DSR e impactan en el acceso a las ILE. Tal como refería previamente, la estrategia implementada por sectores heteropatriarcales a través del reconocimiento de la OC sanitaria y su ejercicio como derecho incólume, guarda en sí un potencial obstaculizador a las ILE y, en consecuencia, sobre su plena efectivización como derecho.

En los escenarios sociopolíticos convergen las influencias tanto de los movimientos feministas que pugnan por reconocimientos de derechos, como de los sectores heteropatriarcales religiosos que buscan sostener la hegemonía de la moral dominante. La reactividad de estos últimos brota ante los avances legales por los cuales los feminismos logran disputar la sexualidad, como eje clave del control social que ejerce el poder religioso (Puga y Vaggione, 2015, pp. 104-105). En este contexto, la religión rescata la construcción política de la OC y la significa como estrategia jurídico-discursiva que incide en el acceso a las ILE (Alegre, 2009, pp. 21-22). La posibilidad de que la objeción moral se traduzca en afectación de derechos es sumamente preocupante en términos de igualdad democrática y de legitimidad del Estado de Derecho, máxime cuando la afectación impacta en los derechos a colectivos que sufren injusticias en términos de redistribución y reconocimiento (Fraser, 2006, pp. 25-39). Por consiguiente, la OC, frente a las ILE, tiene un efecto mucho más público que las objeciones opuestas ante otras obligaciones, lo que la aleja de los preceptos democráticos ${ }^{11}$.

Como bien señala Offe, las sociedades modernas deben enfrentar, en el marco democrático, el problema central de «mantener la diversidad en el interior de la sociedad civil, tratando al tiempo de conseguir en cierta medida, unidad o "ligazón" desde la autoridad política» (Offe, 1992, p. 168). Siguiendo a Jessop (1999, pp. 1-37), cuando se reconoce a la OC como un derecho amplio frente a las ILE, el Estado cristaliza la selectividad estratégica a favor de los sectores heteropatriarcales religiosos que impactan en las acciones jurídicas políticas. De esta manera, es el mismo sistema político normativo el que permite reglamentaciones que, por insuficientes o ausentes, posibilitan un ejercicio abusivo de la OC. En otras palabras, de esta manera el Estado favorece el curso de acción estratégico del sector heteropatriarcal religioso, en desmedro de los derechos de SS/SR.

Interpretar la OC ante las ILE como un derecho de amplio contenido y alcance prima los intereses de las morales religiosas heteropatriarcales sobre los intereses de los colectivos representados por los movimientos feministas. De esta manera, cuando un Estado de Derecho regula ampliamente la OC u omite la concreción de políticas públicas que tiendan a limitarla, está permitiendo la imposición política y burocrática de una moral religiosa y sus técnicas de control sobre los cuerpos "desviados" (Foucault, 2016, pp. 26-28; Jessop, 2007, pp. 109-151). Así, no solo se incumplen las obligaciones internacionales de $\mathrm{DH}$, sino que, a su vez, clausura el desafío de las

\footnotetext{
${ }^{11}$ Para profundizar en las características que la distinguen, recomiendo las lecturas de Alegre, M. (2009). ¿Opresión a conciencia?: La objeción de conciencia en la esfera de la salud sexual y reproductiva. Seminario en Latinoamérica de Teoría Constitucional y Política Papers, paper 66. Recuperado de: http://digitalcommons.law.yale.edu/yls sela/66/ y Triviño Caballero, R. (2014). El peso de la conciencia: la objeción en el ejercicio de las profesiones sanitarias. Madrid, España: CSIC.
} 
democracias modernas de mediar entre "diversidad y unidad" en los desacuerdos de valores (Offe, 1992, p. 168).

Normas que priorizan, por acción u omisión, la OC ante el acceso a las ILE, cristalizan la orientación estatal frente a las fuerzas antagónicas que disputan la conformación de valores de las subjetividades sociopolíticas. Si bien la garantía de la OC como protección a las libertades individuales es trascendente en un Estado democrático, cuando es propuesta ante la SS/SR debe ser contemplada como una excepción y no como una opción del personal sanitario. De lo contrario, el Estado posibilita su ejercicio como una herramienta de presión que confirma el poder de los sectores hetereopatriarcales religiosos. Poder entendido en términos relacionales como «la capacidad de una determinada fuerza para producir un evento que de lo contrario no ocurriría» (Jessop, 2014, p. 28), con influencia en los procesos políticos y en las capacidades del Estado.

\section{Como bien señala Jessop:}

Las estructuras del estado tienen un impacto específico y diferencial sobre la capacidad de las distintas fuerzas políticas para perseguir sus intereses y estrategias particulares en contextos específicos, a través de su control sobre y/o su acceso (in) directo a estas capacidades estatales [capacidades cuya efectividad también depende de los vínculos que establecen con fuerzas y poderes que existen y operan más allá de los limites formales del estado] (Jessop, 2014, p. 31).

La falta de regulación adecuada y la ausencia de sanciones posibilitaron la extensión de la OC sanitaria hasta ser una de las principales barreras de acceso a las ILE (Botero, Cárdenas y Zamberlin, 2019, pp. 139; Diniz, Madeiro y Rosas, 2014, pp. 146-148; Ariza Navarrete y Ramon Michel, 2018, p. 7). El carácter específico y contextual del poder según la perspectiva estratégica relacional (Jessop, 2014, pp. 117), obliga a resaltar el escenario desigual en el que se desarrolla la OC a las ILE, que potencia el impacto obturador de la figura en dicho terreno. Es que las personas usuarias del sistema sanitario se encuentran en situación de subordinación y sujeción respecto a los/as profesionales de la salud. Situación donde "poder y saber" se articulan como control de los cuerpos gestantes: "poder y saber se implican directamente el uno al otro (...), no existe relación de poder sin constitución correlativa de un campo de saber, ni de saber que no suponga y no constituya al mismo tiempo una relación de poder» (Foucault, 2016, p. 28).

Las prácticas de la OC ante las ILE visibilizan la reproducción de estereotipos sexistas y preconceptos religiosos que buscan sostener el poder biomédico y el control sobre los cuerpos gestantes. Es que «en las objeciones se cristalizan un puñado de prejuicios que son simultáneamente médicos, jurídicos y sociales, pero que, al traducirse en el ejercicio unilateral de un desproporcionado poder, la negación a prestar un servicio, tiene un costo excesivamente alto para quienes requieren un(a) ILE» (Botero, Cárdenas y Zamberlin, 2019, p. 145).

Cabe agregar que quienes se encuentran cumpliendo prestaciones sanitarias tienen obligaciones en relación con otras personas determinadas, así como deberes con el interés público en cuanto está en juego el derecho a la salud. Por consiguiente, las obligaciones incumplidas por quienes objetan en estos casos son menos transferibles que los que se encontraban en juego ante las objeciones al servicio militar. Es así que, desde una teoría realista crítica (Jessop, 2007, pp. 109-151), podemos advertir que, cuando la OC no posee una regulación adecuada, las estructuras privilegian las identidades y estrategia de los sectores heteropatriarcales religiosos. Lo expuesto en tanto los efectos de la interposición de la OC a las ILE depende de las definiciones estatales al respecto. 
En este entramado, la religión lejos está de ser el referente de la cohesión social, tal como enuncia Durkheim para las sociedades tradicionales (Giddens, 1999, pp. 183-200). De hecho, tampoco logra sortear el problema del individualismo propio del Estado moderno capitalista, toda vez que la OC es una figura de neto corte liberal que busca proteger las libertades individuales de los/as profesionales de la salud. Por consiguiente, el Estado define y monitorea las fronteras entre las distintas feminidades y masculinidades castigando y clasificando las conductas desviadas de las que no lo son. Es decir, al plantearse la OC como un derecho que es habilitado por el Estado en términos amplios, se establecen cuáles son las conductas y roles en la disputa por el reproductivo de la sexualidad.

El Estado emplea diversos medios de intervención que, en el caso en concreto, se representan en la utilización estratégica del Derecho. Cuando la OC es contemplada como una alternativa legítima de acceso automático, la norma se convierte en la herramienta mediante la cual sectores heteropatriarcales religiosos confirman su poder para interpretar, construir, seleccionar y reproducir, según su propia moral, los cuerpos e identidades. En virtud de lo expuesto podemos sostener que existen formas particulares del patriarcado que están institucionalizadas y son reproducidas por el Estado moderno, tal como sucede en el caso de la OC frente a la SS/SR.

La concepción de la OC a las ILE como un derecho prioritario, naturalmente ligado a los derechos fundamentales, corre el riesgo de transformarse en un recurso contrario a la legitimidad democrática que le ha reconocido (Triviño, 2018, p. 205). Recurso que, a su vez, permitirá la imposición de una moral sobre el derecho a la salud de quienes se encuentran en un estado de sujeción.

5. Algunas consideraciones sobre el alcance de la objeción de conciencia frente a la salud sexual y no reproductiva en términos de democracia e igualdad

Finalmente, las OC en el terreno de la SS/SR nos enfrenta a una cuestión esencialmente conflictiva en nuestras sociedades: la igualdad-desigualdad. En concreto, nos ubica en las desigualdades producto de las diferencias, estereotipos y jerarquizaciones sexo-genéricas.

En el año 2016, el Comité Europeo de Derechos Sociales (CEDS) se pronunció manifestando el impacto discriminatorio de la OC en el terreno de la SS/SR, en particular sobre bases socioeconómicas, territoriales y de género ${ }^{12}$. Cabe resaltar que el Comité de la Convención sobre la Eliminación de toda forma de Discriminación contra la Mujer (Comité CEDAW) establece que los Estados parte deben velar por la salud de las mujeres que se encuentran en circunstancias especialmente difíciles (Comité CEDAW, 1999, p. 4) ${ }^{13}$. Es decir, que la salud de las mujeres debe ser analizada a la luz de la interseccionalidad ${ }^{14}$.

12 Confederazione Generale Italiana del Lavoro (CGIL) v. Italy, Complaint No. 91/2013. (2016, julio). Resolution CM/ResChS(2016). https://hudoc.esc.coe.int/eng/\#5\%22sort\%22:[\%22ESCPublicationDate \%20Descending\%22],\%22ESCD cldentifier\%22:[\%22reschs-2016-3-en\%22]\}

13 Naciones Unidas. (99-02). La Mujer y la Salud. Recomendación General No 24. https://www.acnur.org/fileadmin/Documentos/BDL/2001/1280.pdf

${ }_{14}$ La "interseccionalidad" es un concepto acuñado por el feminismo afroamericano estadunidense incorporado en el discurso académico por Kimberlé Crenshaw en «Demarginalizing the Intersection of Race and Sex: A Black Feminist Critique of Antidiscrimination Doctrine, Feminist Theory and Antiracist Politics», originalmente publicado en la Revista University of Chicago Legal Forum, con la finalidad de cuestionar la tendencia a tratar raza y género como categorías aisladas y hasta excluyentes. De esta manera, ratifica la variedad del colectivo "mujeres", explicando la especial situación de aquellas que se 
Es que el derecho a la salud como hecho social se ve afectado por las posiciones y desigualdades tales como la raza, la etnia y/o el género (Lema, 2015, pp. 293-310). En ese marco, la SS/SR se presenta como una prerrogativa seriamente restringida a causa de numerosos obstáculos jurídicos, procedimentales, prácticos y sociales vinculados de manera directa con múltiples y concomitantes discriminaciones de género, donde la OC tiene un rol estratégico.

La OC, ante las ILE, tiene enormes repercusiones e implica la afectación de derechos de mujeres y personas gestantes, producto de la preservación de ciertas convicciones morales o libertades, por sobre otras. Siguiendo a Nussbaum (2011, p. 81), vivir en democracia significa respetar decisiones con las que no siempre vamos a estar de acuerdo. Por ello, la limitación de la OC ante las ILE pretende respetar los derechos de las personas a elegir estilos de vida y autodeterminación para evitar imposiciones morales. La libertad de conciencia y religiosa, como fundamentos de la OC, son procedentes e incluso deseables, siempre y cuando no afecten, a lo que, en términos de Nussbaum (2011, pp. 76-78), se denomina: el «interés nacional de orden superior», tales como la vida, la salud y la integralidad corporal. La situación de vida, salud e integralidad corporal que se ponen en juego en las ILE pautan los riesgos en torno a la OC, de la misma forma que el orden público cuestionado. Todo ello conduce a la urgencia en la creación de respuestas restrictivas y adecuadas por parte de los Estados. Las divergencias de la figura en el ámbito conducen a la necesidad de una respuesta restrictiva por parte de los Estados.

Si bien la OC, al menos en su concepción clásica, ha aportado al pluralismo democrático, plantearla como una respuesta normalizada en el terreno de la SS/SR posibilita la reproducción e imposición de morales totalizadoras a la vez que pueden quebrantar la legitimidad de las instituciones que facilitan la convivencia colectiva (Triviño, 2018, p. 202). Si en una sociedad democrática habilitamos la posibilidad a determinadas personas de exceptuarse al cumplimiento de específicas cargas legales originadas en su profesión es necesario que esto sea previsto como excepción. Una regulación adecuada de la figura, fijando límites claros y precisos, se presenta como una medida urgente que los Estados de Derecho deben adoptar.

En sociedades plurales, es menester poner el foco en cuan justas son las instituciones que nos inscriben y mediante las cuales socializamos (Miyares, 2003, pp. 12-13). Instituciones socializadoras, como la sanitaria, son la base para democracias más igualitarias donde el reconocimiento debe ser el paradigma. Por ello, la redefinición y análisis de la OC, cuando es interpuesta ante la SS/SR, debe ser hecho bajo el cristal de la perspectiva de género y el enfoque de Derecho. Solo de esta manera se podrán superar las imposiciones morales que reproducen y perpetúan las discriminaciones de género.

En ese marco, los Estados, mediante el Derecho, tienen el deber de regular las relaciones para que sus habitantes logren su realización, pero con un claro eje de ordenar en torno a la inclusión. Estas son las nociones de bien común que una OC abusiva pone en tensión. Cuando los Estados legalizan las interrupciones de embarazo, pero no limitan las estrategias de resistencia susceptibles de obstaculizar, los DSR son forjados como concesiones de las que pueden seleccionarse cuáles merecen respeto y cuáles no. Siguiendo a Miyares (2003, pp. 14-43), la teoría política construye a los sujetos y la cohesión social. Por ello, es importante que cuando se reconoce la libertad y la igualdad, se ponga el foco en las mujeres y las personas

encuentran en la "intersección" de variadas causas de discriminación, que las ubica en una situación de subordinación más gravosa que la acumulación de las mismas (Barrère Unzueta, 2010, pp. 228-235). 
gestantes, ya que no tendremos libertad ni posibilidad de elegir si no hay un verdadero reconocimiento de Derecho, con la correlativa garantía para su ejercicio.

\section{Reflexiones finales}

La acción, reacción e interacción de las fuerzas sociales en juego activan los poderes del Estado. En el caso de la OC en el terreno de la SS/SR y, en concreto, ante ILE, se advierte como ha conjugado la influencia de la religión en el diseño de las políticas públicas y las normas, a punto tal que se da lugar a regulaciones estratégicamente amplias a favor de la procedencia de la OC. Esta selectividad coadyuva a la finalidad ulterior de quienes ejercen la OC como herramienta de control moral sobre los cuerpos gestantes.

En la misma línea, al establecerse normas que, por un lado, reconocen los derechos de SS/SR y, por otro, permiten la OC en términos de derechos sin límite eficaces, el Estado arbitra un sistema funcional en la discriminación de género mediante el que se constituyen categorías de ciudadanos y ciudadanas. De esta manera, los ciudadanos o ciudadanas que exigen la OC motivados en la convicción de que las ILE no deben realizarse, tienen una ciudadanía más efectiva que aquellas personas a las que afectan con su accionar.

En virtud de lo expuesto, se puede afirmar que en el diseño de políticas públicas no tiene la misma repercusión la exigencia de los derechos de SS/SR, que aún son analizados bajo ópticas morales y de excepción o desviación, que las libertades religiosas que pretenden protegerse con la objeción. Cuando la OC es receptada como un derecho con un contenido y alcance amplio e irrestricto, se reconocen y privilegian las fuerzas religiosas que se posiciona más allá del Estado, y se configuran concesiones a determinados sujetos (categoría de ciudadanos y ciudadanas), permitiéndoles exceptuarse legalmente del cumplimiento de una norma. Todo lo cual es funcional al control social, a la confirmación de un poder y a la gubernamentalidad.

Desde esta perspectiva, y siguiendo a Fraser (2006, pp. 25-39), es necesario que la OC, frente a casos de SS/SR, en general, y, en particular, ILE, deba ser ejecutada desde la óptica de políticas de reconocimiento transformadoras de las instituciones y la cultura, para permitir así avanzar en la igualdad, como principio fundamental de una democracia. Es que con la OC en el terreno de la SS/SR se materializan prejuicios médicos, jurídicos y sociales, que al complementarse con el ejercicio de poder/saber de quienes son las personas responsables de prestar los servicios de salud, se traduce en una figura con un excesivo costo para quienes pugnan por reconocimientos y accesos a derechos (Botero, Cárdenas y Zamberlin, 2019, p. 145).

Es imprescindible que, en el marco del Estado de Derecho, se arbitren las medidas y decisiones necesarias para evitar que su ejercicio obstruya el acceso a derechos que tienden a la igualdad. Tomarse en serio la OC significa impedir que un recurso que originariamente promovía la protección de las convicciones personales de las minorías, hoy se traduzca en una imposición de las morales dominantes. De lo contrario «se daría la paradoja de propiciar precisamente aquello contra lo que fue pensado, es decir, situaciones de abuso de poder que perjudiquen a quienes ostentan la posición más débil» (Triviño, 2018, pp. 206-207). 
Bibliografía

Alegre, M. (2009). ¿Opresión a conciencia?: La objeción de conciencia en la esfera de la salud sexual y reproductiva. Seminario en Latinoamérica de Teoría Constitucional y Política, (66), 1-37. Recuperado de: http://digitalcommons.law.yale.edu/yls sela/66/.

Ariza Navarrete, S. y Ramón Michel, A. (2018). Una vuelta de tuerca a la objeción de conciencia: Una propuesta regulatoria a partir de las prácticas del aborto legal en Argentina. Buenos Aires, Argentina: CEDES e IPAS.

Ariza Navarrete, S. (2012). Resistencia al acceso al aborto (no punible): la objeción de conciencia. Revista Derecho Penal, Año I (N²), 23-36. Recuperado de: http://www.saij.gob.ar/doctrina/dacf120189-ariza navarreteresistencias al acceso al.htm.

Barrère Unzueta, M. A. (2010). La interseccionalidad como desafío al mainstreaming de género en las políticas públicas. Revista Vasca de Administración Pública, (8788), 225-252. Recuperado de: https://www.aragon.es/documents/20127/674325/7.\%20Barrere\%20Unzueta, \%2 0M.\%C2\%AAAngeles.pdf/9fb6f5e3-0df2-05dd-664a-06e8fae03a46.

Botero, S., Cárdenas, R. y Zamberlin, N. (2019). ¿De qué está hecha la objeción? Relatos de objetores de conciencia a servicios de aborto legal en Argentina, Uruguay y Colombia. Sexualidad, salud y sociedad. Revista Latinoamericana, (33), 137-157.

Cena, M. J. (2020). Objetar el aborto. En M. Jaime y F. Valdivia (ed.), Mujeres, aborto y religiones en Latinoamérica. Debates sobre política sexual, subjetividades y campo religioso (pp. 149-176). Lima, Perú: Flora Tristán y UNMSM.

Correa, S. y Petchesky, R. (1995). Reproductive and Sexual Rights: A Feminist Perspective. Physis Revista de Saúde Coletiva, 6(1-2),147-177.

Díaz Garcia, E. (2010). Estado de derecho y sociedad democrática. Madrid, España: Taurus.

Diniz, D., Madeiro, A., y Rosas, C. (2014). Conscientious objection, barriers, and abortion in the case of rape: a study among physicians. Brazil. Reproductive Health Matters, 22(43), 141-148. doi: 10.1016/S0968-8080(14)43754-6.

Etchichury, H. J. (2015). El aporte del derecho en el enfoque de derechos. En H.J. Etchichury y M.I. Álvarez (Coord.), Encuentros y (des)encuentros entre las políticas públicas y los derechos sociales (pp. 13-37). Buenos Aires, Argentina: Miño y Dávila.

Foucault, M. (2016). Seguridad, Territorio, Población. Curso en el College de France (1977-1978) ( $1^{a}$ ed., $5^{a}$ reimpresión). Buenos Aires, Argentina: Fondo de Cultura Económica.

Fraser, N. (2006). La Justicia Social en la era de la Política de la Identidad: Redistribución, reconocimiento y participación. En N. Fraser y A. Honneth, ¿Redistribución o reconocimiento? (pp. 17-88). Madrid, España: Traficantes de Sueños.

Gascón Abellán, M. (1990). Obediencia al derecho y objeción de conciencia. Madrid, España: Centro de Estudios Constitucionales.

Giddens, A. (1999). El capitalismo y la moderna teoría social. Barcelona, España: Idea Books.

Jessop, B. (2014). El Estado y el poder. Utopía y praxis latinoamericana, (66), 1-17.

Jessop, B. (2007). Capitalismo(s): Discursos y materialidad en las formaciones sociales capitalistas. Córdoba, Argentina: EDUCC.

Jessop, B. (1999). The Strategic Selectivity of the State: Reflections on a Theme of Poulantzas. Journal of the Hellenic Diaspora, 25 (1-2), 1-37.

Lema Añon, C. (2015). Los desafíos del derecho a la salud: justicia social y condicionantes sociales de la salud. En S. Ribotta, y A. Rossett (ed.), Los 
derechos sociales y su exigibilidad. Libres de temor y miseria (pp. 293-310). Madrid, España: Dykinson.

Miyares, A. (2003). Democracia feminista. Madrid, España: Ediciones Cátedra.

Montaño, S. (1996). Los derechos reproductivos de la mujer. Washington, D.C., EE.UU.: Corte IDH.

Nussbaum, M. C. (2011). Libertad de conciencia: el ataque a la igualdad de respeto. Vivir en democracia implica respetar el derecho de las personas a elegir estilos de vida con los que no estoy de acuerdo (Entrevista de Daniel Gamper Sachse). Madrid, España y Buenos Aires, Argentina: Katz Editores.

Offe, C. (1992). Partidos políticos y nuevos movimientos sociales. Madrid, España: Editorial Sistema.

Peñas Defago, M. A. (2012). Las políticas de salud sexual y reproducción desde un enfoque de derechos humanos. En J.M. Morán Faúndes, M.C. Sgró Ruata y J.M. Vaggione (eds.), Sexualidades, desigualdades y derechos. Reflexiones en torno a los derechos sexuales y reproductivos (pp. 191-223). Córdoba, Argentina: Ciencia, Derecho y Sociedad.

Petracci, M. y Pecheny, M. (2007). Argentina, derechos humanos y sexualidad. Buenos Aires, Argentina: CEDES.

Pietro Sanchis, L. (2011). La objeción de conciencia sanitaria. En M. Gascón Abellán, M. del C. González Carrasco y J. Cantero Martínez (coord.), Derecho Sanitario y bioética. Cuestiones actuales (pp. 981-1011). Valencia, España: Tirant lo Blanch.

Puga, M. y Vaggione, J.M. (2015). La política de la conciencia. La objeción como estrategia contra los derechos sexuales y reproductivos. En M. Vassallo (comp.), Peripecias en la lucha por el derecho al aborto (pp. 94-137). Córdoba, Argentina: Católicas por el Derecho a Decidir.

Rawls, J. (1971). A Theory of Justice (1 $1^{\mathrm{a}}$ ed.). Cambridge: Harvard University Press. Edición mexicana González, M.D. (1979). Teoría de la Justicia. México D.C.: Fondo de Cultura Económica.

Richardson, D. (2000). Constructing sexual citizenship: theorizing sexual Rights. Critical Social Policy, 20(1), 105-135.

Ruiz, A. (2003). El Derecho como discurso y como juego. Revista Jurídica Universidad Interamericana de Puerto Rico, 38, 1-5.

Savulescu, J. (2006). Conscientious objection in medicine. British Medical Journal, 332, 294-297.

Triviño Caballero, R. (2018). Objeción de conciencia. Eunomía. Revista en Cultura de la Legalidad, (15), 198-208. Recuperado de: https://e-revistas. uc3m.es/index.php/EUNOM/article/view/4348.

Triviño Caballero, R. (2014). El peso de la conciencia: la objeción en el ejercicio de las profesiones sanitarias. Madrid, España: CSIC.

Vaggione, J.M. (2012). Introducción. En J.M. Morán Faúndes, M.C. Sgró Ruata y J.M. Vaggione (Eds.), Sexualidades, desigualdades y derechos. Reflexiones en torno a los derechos sexuales y reproductivos (pp. 13-55). Córdoba, Argentina: Ciencia, Derecho y Sociedad. 